\title{
The Shear Viscosity to Entropy Density Ratio of Trapped Fermions in the Unitarity Limit
}

\author{
T. Schäfer \\ Department of Physics, North Carolina State University, Raleigh, NC 27695
}

\begin{abstract}
We extract the shear viscosity to entropy density ratio $\eta / s$ of cold fermionic atoms in the unitarity limit from experimental data on the damping of collective excitations. We find that near the critical temperature $\eta / s$ is roughly equal to $1 / 2$ in units of $\hbar / k_{B}$. With the possible exception of the quark gluon plasma, this value is closer to the conjectured lower bound $1 /(4 \pi)$ than any other known liquid.
\end{abstract}

\section{INTRODUCTION}

Strongly correlated quantum systems play an important role in many different areas of physics. Systems of interest in atomic, condensed matter, and nuclear physics span many orders of magnitude in energy scale but exhibit a number of universal properties. Recently there has been renewed interest in the transport properties of strongly correlated systems. Experiments at the Relativistic Heavy Ion Collider (RHIC) indicate that at temperatures close to the critical temperature $T_{c}$ the quark gluon plasma is strongly interacting. The strongly interacting quark gluon plasma (sQGP) is characterized by a very small shear viscosity to entropy ratio, a small heavy quark diffusion coefficient, and a large opacity for high energy jets [1, 2, 3, 4, 5].

From a theoretical point of view not much is known about transport coefficients of strongly correlated systems. If the interaction is weak then the mean free path and the shear viscosity are large. As the strength of the interaction increases the mean free path and the viscosity drop but there are good reasons to believe that the shear viscosity always remains finite. Kovtun et al. conjectured that there is a universal lower bound $\eta / s \geq \hbar /\left(4 \pi k_{B}\right)[6]$. Here, $\eta$ is the shear viscosity, $s$ is the entropy density, $\hbar$ is Planck's constant and $k_{B}$ is the Boltzmann constant. The bound is saturated in the case of strongly coupled gauge theories that have a dual description in terms of a gravitational theory.

In this work we test the viscosity bound conjecture by extracting $\eta / s$ from experimental data on the damping of collective oscillations of a cold atomic gas near a Feshbach resonance. Cold atomic gases provide an ideal system to test the conjecture because both the temperature and the interaction can be continuously adjusted. Also, because of universality, atoms in the unitarity limit are equivalent to other Fermi liquids with a large scattering length like dilute neutron matter. Collective modes in the atomic system have been studied in a number of experiments [7, 8, 9, 10]. In the weak coupling regime the frequency and damping constant of collective modes can be understood in terms of the Boltzmann equation. In the unitarity limit the frequency of collective modes is well described by ideal hydrodynamics [11, 12, 13]. In the present work we include viscous corrections and use experimental and quantum Monte Carlo data on the thermodynamics to extract $\eta / s$. Previous studies of damping near the unitarity limit can be found in [14, 15, 16].

\section{EULER HYDRODYNAMICS}

We shall assume that the system is approximately described by ideal (Eulerian) fluid dynamics. The equation of continuity and of momentum conservation are given by

$$
\begin{aligned}
\frac{\partial n}{\partial t}+\vec{\nabla} \cdot(n \vec{v}) & =0, \\
m n \frac{\partial \vec{v}}{\partial t}+m n(\vec{v} \cdot \vec{\nabla}) \vec{v} & =-\vec{\nabla} P-n \vec{\nabla} V,
\end{aligned}
$$

where $n$ is the number density, $m$ is the mass of the atoms, $\vec{v}$ is the fluid velocity, $P$ is the pressure and $V$ is the external potential. The trapping potential is approximately harmonic

$$
V=\frac{m}{2} \sum_{i} \omega_{i}^{2} r_{i}^{2}
$$

In the unitarity limit the equation of state at zero temperature is a simple polytrope $P \sim n^{\gamma+1}$ with $\gamma=2 / 3$. At finite temperature the equation of state is more complicated, but universality implies that the isentropic compressibility is unaffected,

$$
\left(\frac{\partial P}{\partial n}\right)_{S}=(\gamma+1) \frac{P}{n} .
$$

The equilibrium distribution $n_{0}$ can be determined from the hydrostatic equation $\vec{\nabla} P_{0}=-n_{0} \vec{\nabla} V$. At $T=0$

$$
n_{0}(\vec{r})=n_{0}(0)\left(1-\sum_{i} \frac{r_{i}^{2}}{R_{i}^{2}}\right)^{1 / \gamma}, \quad R_{i}^{2}=\frac{2 \mu}{m \omega_{i}^{2}},
$$

where $\mu$ is the chemical potential. In the unitarity limit the chemical potential is related to the Fermi energy as $\mu=\xi E_{F}$, where $\xi$ is a universal parameter $(\xi \simeq 0.44$ according to the quantum Monte Carlo calculation [17]). The central density and the total number of particles are

$$
n_{0}(0)=\frac{1}{3 \pi^{2}}\left(\frac{2 m \mu}{\xi}\right)^{3 / 2}, \quad N=\frac{1}{3 \xi^{3 / 2}}\left(\frac{\mu}{\bar{\omega}}\right)^{3},
$$




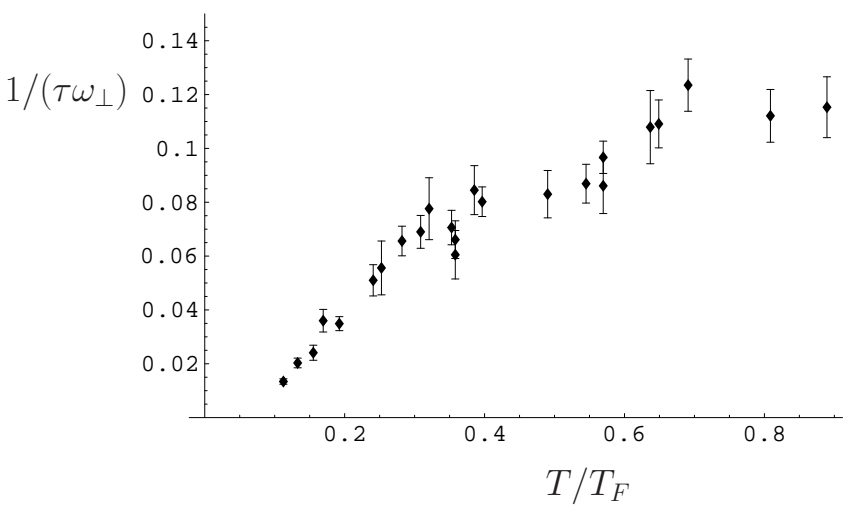

FIG. 1: Temperature dependence of the damping rate for the radial breathing mode of a trapped ${ }^{6} \mathrm{Li}$ gas near the Feshbach resonance at $840 \mathrm{G}$, from Kinast et al. 8]. Here $\tau$ is the damping time and $\omega_{\perp}$ is the radial trap frequency. We have used the calibration curve in [18] to convert the $\tilde{T}$ scale of [8] to $T / T_{F}$.

where $\bar{\omega}=\left(\omega_{1} \omega_{2} \omega_{3}\right)^{1 / 3}$. Consider small oscillations $n=n_{0}+\delta n$. From the linearized continuity and Euler equation we get [11]

$$
m \frac{\partial^{2} \vec{v}}{\partial t^{2}}=-\gamma(\vec{\nabla} \cdot \vec{v})(\vec{\nabla} V)-\vec{\nabla}(\vec{v} \cdot \vec{\nabla} V),
$$

where we have dropped terms of the form $\nabla_{i} \nabla_{j} \vec{v}$ that involve higher derivatives of the velocity. Using a scaling ansatz $v_{i}=a_{i} x_{i} \exp (i \omega t)$ (no sum over $i$ ) we get

$$
\left(2 \omega_{j}^{2}-\omega^{2}\right) a_{j}+\gamma \omega_{j}^{2} \sum_{k} a_{k}=0 .
$$

This is a simple linear equation of the form $M a=0$. Nontrivial solutions correspond to $\operatorname{det}(M)=0$. In the case of a trapping potential with axial symmetry, $\omega_{1}=\omega_{2}=\omega_{0}$, $\omega_{3}=\lambda \omega_{0}$, we get $\omega^{2}=2 \omega_{0}^{2}$ and [11, 12, 13]

$$
\begin{aligned}
\omega^{2}= & \omega_{0}^{2}\left\{\gamma+1+\frac{\gamma+2}{2} \lambda^{2}\right. \\
& \left. \pm \sqrt{\frac{(\gamma+2)^{2}}{4} \lambda^{4}+\left(\gamma^{2}-3 \gamma-2\right) \lambda^{2}+(\gamma+1)^{2}}\right\} .
\end{aligned}
$$

In the unitarity limit $(\gamma=2 / 3)$ and for a very asymmetric trap, $\lambda \rightarrow 0$, the eigenfrequencies are $\omega^{2}=2 \omega_{0}^{2}$ and $\omega^{2}=(10 / 3) \omega_{0}^{2}$. The mode $\omega^{2}=(10 / 3) \omega_{0}^{2}$ is a radial breathing mode with $\vec{a}=(a, a, 0)$ and the mode $\omega^{2}=2 \omega_{0}^{2}$ corresponds to a radial quadrupole $\vec{a}=(a,-a, 0)$.

\section{VISCOUS CORRECTIONS}

The energy dissipated due to viscous effects is

$$
\begin{aligned}
\dot{E}= & -\frac{1}{2} \int d^{3} x \eta(x)\left(\partial_{i} v_{j}+\partial_{j} v_{i}-\frac{2}{3} \delta_{i j} \partial_{k} v_{k}\right)^{2} \\
& -\int d^{3} x \zeta(x)\left(\partial_{i} v_{i}\right)^{2},
\end{aligned}
$$

where $\eta$ is the shear viscosity and $\zeta$ is the bulk viscosity. In the unitarity limit the system is scale invariant and the bulk viscosity in the normal phase vanishes. The situation is more complicated in the superfluid phase. In this case the normal and superfluid components can flow independently and in addition to the shear viscosity there are three bulk viscosities $\zeta_{i}$. Two of the three bulk viscosities, $\zeta_{1}$ and $\zeta_{2}$, can be shown to vanish as a consequence of scale invariance [19]. The third bulk viscosity, $\zeta_{3}$, only contributes to dissipation if the normal components is moving relative to the superfluid [20]. In the following we shall consider modes in which the two components move in phase and ignore bulk viscosity. We will also assume that the viscosity only depends on $x$ through the local density and temperature. This is valid if the density and temperature are varying slowly, and is consistent with the local density approximation for the density profile. We note that in this approximation the equation of state and the transport coefficients reflect the conformal invariance of the microscopic dynamics, even though scale invariance is broken by the external potential.

For the radial scaling flows given in equ. (9) we have

$$
\overline{\dot{E}}=-\frac{2}{3}\left(a_{x}^{2}+a_{y}^{2}-a_{x} a_{y}\right) \int d^{3} x \eta(x),
$$

where $\bar{E}$ is a time average. The damping rate is determined by the ratio of the energy dissipated to the total energy of the collective mode. The kinetic energy is

$$
E_{k i n}=\frac{m}{2} \int d^{3} x n(x) \vec{v}^{2}=\frac{m N}{2}\left(a_{x}^{2}+a_{y}^{2}\right)\left\langle x^{2}\right\rangle .
$$

At $T=0$ we find $\left\langle x^{2}\right\rangle=R_{\perp}^{2} / 8$, where $R_{\perp}$ is the transverse size of the cloud. At non-zero temperature we can use the Virial theorem [21] to relate $\left\langle x^{2}\right\rangle$ to the total energy of the equilibrium state, $\left\langle x^{2}\right\rangle /\left\langle x^{2}\right\rangle_{T=0}=E / E_{T=0}$. The damping rate is 15,22 ]

$$
-\frac{1}{2} \frac{\bar{E}}{E}=\frac{2}{3} \frac{a_{x}^{2}+a_{y}^{2}-a_{x} a_{y}}{a_{x}^{2}+a_{y}^{2}} \frac{\int d^{3} x \eta(x)}{m N\left\langle x^{2}\right\rangle} .
$$

Note that the second factor on the RHS is $1 / 2$ for the radial breathing mode and $3 / 2$ for the radial quadrupole mode. If this dependence could be demonstrated experimentally, it would confirm that the damping is indeed dominated by shear stress. Another possibility is to compare the breathing mode with a scissors mode. The scissors mode is characterized by the velocity field $\vec{v}=a \vec{\nabla}(x y)$. The frequency is $\omega^{2}=\omega_{x}^{2}+\omega_{y}^{2}$ and the second factor in equ. (13) is 6 .

For the unitary Fermi gas the ratio of the shear viscosity to the entropy density is given by universal function that depends only on the ratio $T / T_{F}, \eta(\mu, T)=$ $\alpha\left(T / T_{F}\right) s(\mu, T)$. Here, the Fermi temperature is given by $T_{F}=\left(3 \pi^{2} n\right)^{2 / 3} /(2 m)$. In the local density approximation this implies that $\eta(x)=\alpha\left(T / T_{F}(x)\right) s(x)$, where $T_{F}(x)$ is the local Fermi temperature. We shall assume 


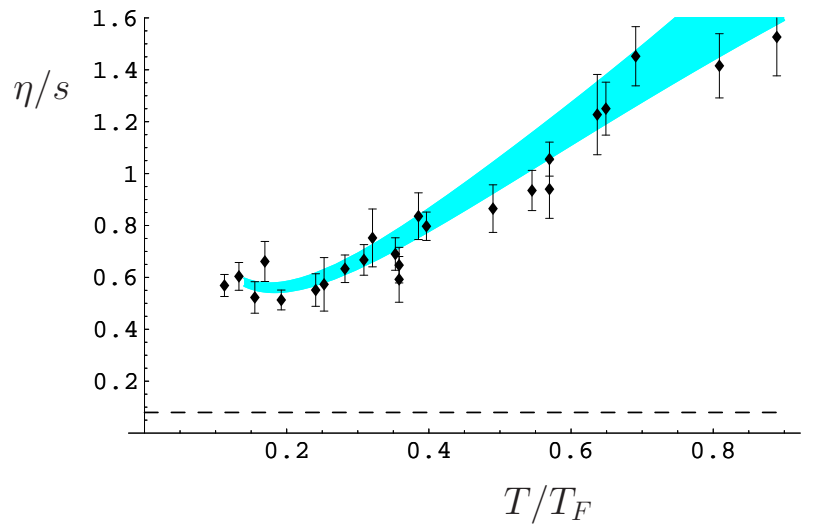

FIG. 2: Viscosity to entropy density ratio of a cold atomic gas in the unitarity limit. This plot is based on the damping data published in [8] and the thermodynamic data in [18, 23]. The dashed line shows the conjectured viscosity bound $\eta / s=$ $1 /(4 \pi)$. The shaded band is a systematic error estimate based on the contribution to $\dot{E}$ from atoms outside a surface at optical depth one.

that $\alpha$ is a smooth function and replace $T_{F}(x)$ by its value at the center of the trap. This approximation can be checked a posteriori. We note that since the flow profile has a simple scaling form the damping rate is proportional to the volume integral of the shear viscosity. If $\eta \sim s$ then the damping rate scales with the total entropy. The kinetic energy, on the other hand, is proportional to the number of particles. The shear viscosity to entropy density ratio extracted from the radial breathing mode is

$$
\frac{\eta}{s}=\frac{3}{4} \xi^{1 / 2}(3 N)^{1 / 3}\left(\frac{\bar{\omega} \Gamma}{\omega_{\perp}^{2}}\right)\left(\frac{E}{E_{T=0}}\right)\left(\frac{N}{S}\right),
$$

where $\Gamma / \omega_{\perp}=1 /\left(\tau \omega_{\perp}\right)$ is the dimensionless damping rate.

\section{BREAKDOWN OF HYDRODYNAMICS}

Near the edge of the cloud the mean free path is comparable to the characteristic length scale over which the velocity field varies and the hydrodynamic description breaks down. In this regime the density is low and the mean free path can be estimated using kinetic theory. In the limit $n \ll(m T)^{3 / 2}$ the mean free path is given by [16]

$$
l_{m f p}=(n \sigma)^{-1}, \quad \sigma=\frac{4 \pi}{3} \frac{1}{m k_{B} T} .
$$

Following [22] we can define a surface $r_{0}(\theta)$ by the condition that a particle incident from the exterior of the cloud has a chance of no larger than $1 / e$ of colliding with another particle

$$
1=\int_{r_{0}(\theta)}^{\infty} \frac{d s}{l_{m f p}(\vec{r})}
$$

Here, $\theta$ denotes the angle between $d s$ and the $z$-axis. Ideally, the hydrodynamic description inside the surface $r_{0}(\theta)$ should be coupled to kinetic theory outside this surface. In this work we shall be less ambitious and use equ. (16) in order to estimate the systematic uncertainty in our determination of $\eta / s$. For this purpose we compute the contribution to $\dot{E}$ in equ. (13) that comes from atoms outside $r_{0}(\theta)$ and treat it as a systematic error in the damping rate.

\section{THERMODYNAMICS}

Experimental results for $\Gamma / \omega$ are shown in Fig. (11). In order to extract $\eta / s$ we also need information on the entropy per particle. In the unitarity limit there are only two energy scales in the problem, the chemical potential $\mu$ and the thermal energy $k_{B} T$ (in the following we will set $k_{B}$ to one). The associated density scales are $n_{f}(\mu)$ and $\lambda_{T}^{-3}$, where $n_{f}(\mu)=(2 m \mu)^{3 / 2} /\left(3 \pi^{2}\right)$ is the density of a free Fermi gas and $\lambda_{T}=(2 \pi m T)^{-1 / 2}$ is the thermal wave length. All thermodynamic quantities can be expressed as suitable powers of either $n_{f}(\mu)$ or $\lambda_{T}^{3}$ times a function of the dimensionless quantity $y=T / \mu$. For example, we can write the pressure as 24]

$$
P(\mu, T)=\frac{2}{5} \mu n_{f}(\mu) \mathcal{G}(y)=\frac{2}{5} T \lambda_{T}^{-3} \mathcal{W}\left(y^{-1}\right),
$$

where the first form is more useful at small $T$ and the second at high $T$. Using standard thermodynamic identities one can show that

$$
\begin{aligned}
n(\mu, T) & =n_{f}(\mu) \mathcal{F}(y), \\
s(\mu, T) & =\frac{2}{5} n_{f}(\mu) \mathcal{G}^{\prime}(y),
\end{aligned}
$$

where $n$ is the density, $s$ is the entropy density, and $\mathcal{F}(y)=\mathcal{G}(y)-2 y \mathcal{G}^{\prime}(y) / 5$. At $T=0$ the function $\mathcal{G}(y)$ is related to the parameter $\xi$ introduced above, $\mathcal{G}(0)=\xi^{-3 / 2}$. The functions $\mathcal{G}(y), \mathcal{F}(y)$ refer to the bulk system. Trapped systems can be described using the local density approximation. Experiments typically involve $10^{5}-10^{6}$ atoms and the local density approximation is very accurate. The density of the trapped system is

$$
n_{0}(x)=n(\mu-V(x), T),
$$

where $V(x)$ is the trapping potential. Similar relations hold for the energy and entropy density. For $T=0$ equ. (20) reduces to equ. (5).

The function $\mathcal{G}(y)$ can be extracted from quantum Monte Carlo data or from calorimetric experiments with trapped fermions. A number of Monte Carlo calculations have appeared over the last couple of years [25, 26, 27], but there are still significant disagreements between the results. Burovski et al. find $T_{c} / T_{F}=0.152(7)$ and a critical entropy per particle $S / N=0.16(2)$ 26]. Bulgac et al. quote $T_{c} / T_{F}=0.23(2)$ and $S / N \simeq 1.1$ [27]. 
Using the local density approximation the results of Bulgac et al. correspond to a critical entropy per particle of $S / N \simeq 2.1$ for the trapped system. The reason that the entropy per particle is larger in a trapped system is that the density near the edges is smaller, and therefore the dimensionless temperature $m T / n^{2 / 3}$ larger.

The Duke group has performed a series of calorimetric measurements [18, 23]. Kinast et al. [18] provide a simple parametrization of the energy of the trapped system as a function of $t=T / T_{F}$. The result is

$$
E=E_{0} \begin{cases}1+97.3 t^{3.73} & t<t_{c} \\ 1+4.98 t^{1.43} & t>t_{c}\end{cases}
$$

with $E_{0}=0.53 E_{F}$ and $T_{c} / T_{F}=0.29(2)$. Luo et al. 23] give a similar parametrization of the entropy,

$$
S / N= \begin{cases}4.6\left(e-e_{0}\right)^{0.61} & e<e_{c} \\ 4.0\left(e-e_{0}\right)^{0.45} & e>e_{c}\end{cases}
$$

where $e=E / E_{F}$. The critical entropy per particle is $S / N=2.7$, roughly compatible with the Monte Carlo results of Bulgac et al., but significantly larger than the results of Burovski et al.

\section{RESULTS AND CONCLUSIONS}

In Fig. 2 we show $\eta / s$ extracted using both the damping data and the calorimetry from the Duke group. We observe that $\eta / s$ is small $(\sim 0.5)$ near $T_{c}$ and slowly grows with temperature for $T>T_{c}$. The value of $\eta / s$ near $T_{c}$ is about six times larger than the conjectured viscosity bound and consistent with the picture of a very strongly correlated liquid. Indeed, the extracted value $\eta / s \sim 0.5$ is smaller than the previously known minimum for all other liquids, $\eta / s \sim 0.7$ for liquid Helium near the lambda point [6]. Even smaller values of $\eta / s$ have been reported for the quark gluon plasma produced at RHIC, but the uncertainties remain large [2, 28]. We note that the systematic uncertainty near the minimum is small, but that it increases as a function of temperature.

There are a number of issues that need to be addressed in more detail. We argued that it is important to establish that shear viscosity is indeed the dominant damping mechanism. This can be done either by studying the dependence of the damping time on the type of collective mode, or by studying the dependence on system size. Kinast et al. collected some data on system size dependence below $T_{c}$ and find that $\Gamma / \omega_{\perp}$ is roughly independent of the number of particles [8]. This is not consistent with the scaling in equ. (14). Data on system size dependence can also be used to determine at what point hydrodynamics is breaking down.

It is also important to understand the damping mechanism below $T_{c}$ in more detail. The data show a very simple linear behavior in the variable $\tilde{T} \sim\left(T / T_{F}\right)^{2 / 3}$. The natural framework for understanding the damping mechanism in the regime below $T_{c}$ is superfluid (two-fluid) hydrodynamics [20]. There are several sound modes in superfluid hydrodynamics. First sound is an excitation in which the superfluid and normal components move together, whereas second sound corresponds to an oscillation of the superfluid component against the normal one. The damping of first sound is governed by the shear stress of the normal component. It is likely that the collective excitations that have been observed experimentally are ordinary (first) sound modes, but it is not obvious why the damping constant is linear in $\tilde{T}$. We should note that a linear behavior was observed in trapped Bose gases [29], where it was attributed to Landau damping by normal excitations [30].

Finally, there are a number of technical aspects of our analysis that should be improved. We have assumed that the quantity $\alpha=\eta / s$ is only weakly temperature dependent. Near the the minimum of $\eta / s$ this is a good approximation, but at higher temperature the uncertainty inherent in this approximation grows, as does the uncertainty related to the breakdown of hydrodynamics near the the surface of the cloud. In this regime a Boltzmann description should be used. In the present work we have neglected dissipation due to temperature gradients and thermal conductivity. This is expected to be a good approximation for scaling flows because oscillations in density are proportional to the equilibrium density $\delta n(x) \sim n_{0}(x)$. For isentropic oscillations $\delta T \sim(\delta n / n) T$ and to leading order no temperature gradients are present.

Acknowledgments: This work is supported in part by the US Department of Energy grant DE-FG0203ER41260. I would like to thank Dam Son for useful correspondence, and John Thomas and Andrey Turlapov for pointing out an error in an earlier draft.
[1] I. Arsenne et al. [Brahms], B. Back et al. [Phobos], K. Adcox et al. [Phenix], J. Adams et al. [Star], "First Three Years of Operation of RHIC", Nucl. Phys. A757, 1-183 (2005).

[2] D. Teaney, Phys. Rev. C 68, 034913 (2003) nucl-th/0301099.

[3] E. Shuryak, Prog. Part. Nucl. Phys. 53, 273 (2004) hep-ph/0312227.
[4] M. Gyulassy and L. McLerran, Nucl. Phys. A 750, 30 (2005) nucl-th/0405013.

[5] U. W. Heinz, preprint, nucl-th/0512051

[6] P. Kovtun, D. T. Son and A. O. Starinets, Phys. Rev. Lett. 94, 111601 (2005) hep-th/0405231.

[7] J. Kinast, S. L. Hemmer, M. E. Gehm, A. Turlapov, and J. E. Thomas, Phys. Rev. Lett. 92, 150402 (2004).

[8] J. Kinast, A. Turlapov, J. E. Thomas, Phys. Rev. Lett. 
94, 170404 (2005) cond-mat/0502507.

[9] M. Bartenstein, A. Altmeyer, S. Riedl, S. Jochim, C. Chin, J. Hecker Denschlag, and R. Grimm, Phys. Rev. Lett. 92, 203201 (2004) cond-mat/0412712.

[10] A. Altmeyer, S. Riedl, C. Kohstall, M. Wright, R. Geursen, M. Bartenstein, C. Chin, J. Hecker Denschlag, R. Grimm, preprint, cond-mat/0609390

[11] H. Heiselberg, Phys. Rev. Lett. 93, 040402 (2004) cond-mat/0403041.

[12] S. Stringari, Europhys. Lett. 65, 749 (2004) cond-mat/0312614.

[13] A. Bulgac and G. F. Bertsch, Phys. Rev. Lett. 94, 070401 (2005) cond-mat/0404687.

[14] P. Massignan, G. M. Bruun, H. Smith, Phys. Rev. A 71, 033607 (2005) cond-mat/0409660.

[15] B. A. Gelman, E. V. Shuryak, and I. Zahed, Phys. Rev. A 72, 043601 (2005) nucl-th/0410067.

[16] G. M. Bruun, H. Smith, Phys. Rev. A 75, 043612 (2007) cond-mat/0612460.

[17] J. Carlson, S. Y. Chang, V. R. Pandharipande, K. E. Schmidt, Phys. Rev. Lett. 91, 50401 (2003).

[18] J. Kinast, A. Turlapov, J. E. Thomas, Q. Chen, J. Stajic, and K. Levin, Science 307, 1296 (2005) cond-mat/0502087.

[19] D. T. Son, Phys. Rev. Lett. $98020604 \quad$ (2007) cond-mat/0511721.

[20] I. M. Khalatnikov, An Introduction to the Theory of Superfluidity, W. A. Benjamin, Inc. (1965).

[21] J. E. Thomas, J. Kinast, A. Turlapov, Phys. Rev. Lett. 95, 120402 (2005) cond-mat/0503620.

[22] G. M. Kavoulakis, C. J. Pethick, H. Smith, Phys. Rev. A 57, 2938 (1998) cond-mat/9710130.

[23] L. Luo, B. Clancy, J. Joseph, J. Kinast, J. E. Thomas, preprint, cond-mat/0611566

[24] T.-L. Ho, Phys. Rev. Lett. 92, 090402 (2004) cond-mat/0309109.

[25] D. Lee and T. Schäfer, Phys. Rev. C 73, 015202 (2006) nucl-th/0509018.

[26] E. Burovski, N. Prokof'ev, B. Svistunov, and M. Troyer, Phys. Rev. Lett. 96, 160402 (2006), cond-mat/0602224.

[27] A. Bulgac, J. Drut, and P. Magierski, Phys. Rev. Lett. 96, 090404 (2006) cond-mat/0505374; Int. J. Mod. Phys. B 20, 5165 (2006) cond-mat/0602091.

[28] T. Hirano and M. Gyulassy, Nucl. Phys. A 769, 71 (2006) nucl-th/0506049.

[29] F. Chevy, V. Bretin, P. Rosenbusch, K. W. Madison, and J. Dalibard, Phys. Rev. Lett. 88, 250402 (2002).

[30] P. O. Fedichev, G. V. Shlyapnikov, and J. T. M. Walraven, Phys. Rev. Lett. 80, 2269 (1998). 